\title{
On Cartesian Products of Good Lattices
}

\author{
By S. K. Zaremba
}

\begin{abstract}
Good lattices yield a powerful method of computing multiple integrals. Asymptotically, a lattice generated by one good lattice point is much more efficient than a Cartesian product of such lattices. However, if the number of dimensions is large, this does not always apply to the case when the number of points remains within reasonable limits. Examples of such products of two or three lattices being more efficient than good lattices generated by single lattice points are systematically presented. Additional symmetries of Cartesian products of lattices offer a further advantage when the integrand has to be symmetrized beforehand.
\end{abstract}

1. Good lattices are known as a most efficient tool for computing numerically multiple integrals (see, for instance, [1], [3], [7]). Given the s-dimensional unit interval

$Q^{s:} \quad 0 \leqslant x_{i} \leqslant 1 \quad(i=1, \ldots, s)$,

we choose an integer $m$, described as the modulus, and a lattice point $\mathbf{g}$, that is, a point or, equivalently, a vector with integral coordinates. Throughout, the coordinates of a point will be denoted by a letter with subscripts going from 1 to $s$, the point itself being denoted by the same letter in bold face. We put

$$
R(\mathbf{h})=\max \left(1,\left|h_{1}\right|\right) \cdots \max \left(1,\left|h_{s}\right|\right)
$$

and denote by $\rho(\mathrm{g})$ the minimum of $R(\mathrm{~h})$ over all lattice points $\mathbf{h} \neq \mathbf{0}=\langle 0, \ldots, 0\rangle$ satisfying

$$
\mathbf{g} \cdot \mathbf{h} \equiv \mathbf{0} \quad(\bmod m)
$$

where the dot denotes a scalar product. Following Hlawka [1], $\mathrm{g}$ is described as a good lattice point modulo $m$ if $\rho(\mathrm{g})$ is sufficiently large in relation to $m$.

Given a function $f$ defined over $Q^{s}$, we take, as the approximate value of $\int_{Q^{s}} f(\mathbf{x}) d \mathbf{x}$, the expression

$$
m^{-1} \sum_{k=0}^{n-1} f\left(m^{-1} k \mathrm{~g}\right),
$$

where the coordinates of $\mathrm{m}^{-1} \mathrm{~kg}$ are reduced modulo 1 . This reduction becomes unnecessary if we think of $f$ as being represented by a multiple Fourier series

$$
\sum_{\mathbf{h} \in \mathbf{Z}^{s}} c_{\mathbf{h}} \exp (2 \pi i \mathbf{h} \cdot \mathbf{x}) \text {. }
$$

If, for some integer $r \geqslant 1$ : (i) The mixed partial derivative

Received May 30, 1975.

AMS (MOS) subject classifications (1970). Primary 65D30; Secondary 10B99, 65 A05. 


$$
\frac{\partial^{r s} f}{\partial x_{1}^{r} \cdots \partial x_{s}^{r}}
$$

is of bounded variation in the sense of Hardy and Krause over $Q^{s}$, and (ii) the values of $f$ and, when $r \geqslant 2$, of its partial derivatives up to $\partial^{(r-1) s} f /\left(\partial x_{1}^{r-1} \cdots \partial x_{s}^{r-1}\right)$ agree on opposite sides of $Q^{s}$; then

$$
\left|c_{\mathrm{h}}\right| \leqslant K_{r} R(\mathrm{~h})^{-(r+1)},
$$

where $K_{r}$ depends on the total variations of (4) in the sense of Vitali over $Q^{s}$ and its faces [7].

It follows easily that under such circumstances the absolute value of the error of integration is bounded by

$$
K_{r} P^{(r+1)}(\mathrm{g})
$$

where, for any $n \geqslant 2, P^{(n)}(\mathrm{g})=\Sigma R(\mathrm{~h})^{-n}$, this sum being extended to all lattice points $\mathbf{h} \neq \mathbf{0}$ which satisfy (1). It has been proved [7] that for any sufficiently large modulus $m$ and any lattice point $\mathbf{g}$,

$$
P^{(n)}(\mathrm{g}) \leqslant \frac{2^{3 s+1}(\log m)^{s-1}}{(s-1) !(\log 2)^{s-1} \rho(\mathrm{g})^{n}},
$$

and [8] that for any sufficiently large $m$ there exist lattice points $g$ modulo $m$ satisfying

$$
\rho(\mathrm{g})>(s-1) ! m /(2 \log m)^{s-1} .
$$

However, numerical evidence ([4], [2]) suggests that $P^{(n)}(\mathrm{g})=O\left(\rho(\mathrm{g})^{-n} \log m\right)$, and that for sufficiently large $m$, there exist lattice points with $\rho(\mathrm{g})$ of the order of $m /(\log m)^{s-2}$; this has been proved for $s=2$ (see, for instance, [6]).

2. In two dimensions, lattice points with the best possible ratio $\rho(\mathrm{g}): m$ are constructed with Fibonacci numbers [6], but in a higher number of dimensions, a laborious search for good lattice points is necessary. The first tables of good lattice points were produced by A. I. Saltykov [5] following a method devised by Korobov; they are reproduced in an appendix to a book [3] by the latter, and also in [4, pp. 158-165], where the corresponding values of $P^{(2)}$ are shown as well. It should be noted, though, that these lattice points, in three to ten dimensions, were obtained by a suboptimization procedure; more precisely:

(1) The set of numbers considered as possible moduli was restricted to primes, or, above 10007, to products of two large primes; in fact, later computations indicated that, on the contrary, the most favorable moduli were, with very few exceptions, composite numbers, and, more particularly, numbers having at least one small divisor.

(2) The lattice points were restricted to the type of $\left\langle 1, a, \ldots, a^{s-1}\right\rangle$ in the case of prime moduli, and to the type of

$$
\left\langle p_{1}+p_{2}, p_{1} b+p_{2} a, \ldots, p_{1} b^{s-1}+p_{2} a^{s-1}\right\rangle
$$

when the modulus was a product $p_{1} p_{2}$ of two primes. 
(3) The optimized function was neither $\rho(\mathrm{g})$ nor $P^{(n)}(\mathrm{g})$ for any particular choice of $n$, but, in the case of a prime modulus, a function $H(a)$ such that $H(a)-1$ was of the same order of magnitude as $P^{(2)}(\mathrm{g})$ without, however, being exactly proportional to it. When the modulus was a product of two primes, the connection between the minimized function and $P^{(2)}(\mathrm{g})$ was less obvious.

Tables of good lattice points in three and four dimensions based on the optimization of $\rho(\mathrm{g})$ with $\mathbf{g}=\left\langle 1, g_{2}, \ldots, g_{s}\right\rangle$ were compiled by Dominique Maisonneuve [4] . An additional table of good lattice points in three dimensions with moduli ranging from 2120 to 6066 [2] was published by Gershon Kedem and the present author following the same principles. Both sets of tables show the values of $P^{(2)}(\mathrm{g})$ and $P^{(4)}(\mathrm{g})$ for each g. For two-dimensional Fibonacci lattices, a short table of values of $P^{(2)}(\mathrm{g})$ can be found in [9].

3. The advantages of the method of good lattice points for the computation of multiple integrals are obvious from what was said above, but the condition (ii) of "periodicity" imposed on the integrand may seem to point to a serious limitation of the method. However, there are ways (see, for instance [3, pp. 52-63], or [7, pp. 55-65]) of transforming the integrand so as to satisfy this condition without affecting the value of the integral. Methods involving changes of variables greatly increase the variation of the partial derivatives of the integrand over $Q^{s}$, and, therefore, also the value of the coefficient $K_{r}$ in the upper bound of the error of integration [7, pp. 58-59]; for this reason, the present author would not recommend the use of such methods. The simplest method, which is probably the only practical one in the case of a very large number of dimensions, consists of symmetrizing the integrand $f(\mathbf{x})$, that is, replacing it by the function

$$
\begin{array}{r}
F(\mathbf{x})=2^{-s}\left\{f\left(x_{1}, \ldots, x_{s}\right)+f\left(1-x_{1}, x_{2}, \ldots, x_{s}\right)\right. \\
+f\left(x_{1}, 1-x_{2}, x_{3}, \ldots, x_{s}\right)+f\left(1-x_{1}, 1-x_{2}, x_{3}, \ldots, x_{s}\right) \\
\left.\quad+\cdots+f\left(1-x_{1}, 1-x_{2}, \ldots, 1-x_{s}\right)\right\}
\end{array}
$$

if $f$ satisfies (i) with $r \geqslant 1, F$ satisfies (i) and (ii) with $r=1$.

So far, nobody seems to have noticed that when we use this method with good lattice points (or, for that matter, not so good ones), the number of values of $f$ to be computed can be halved, owing to a symmetry in the lattice generated by a lattice point modulo $m$. Indeed, the congruence

$$
(m-k) m^{-1} g_{i} \equiv 1-k m^{-1} g_{i}(\bmod 1) \quad(i=1, \ldots, s)
$$

is not affected by the reduction of the coordinates modulo 1 . Consequently, whenever the lattice reduced modulo 1 in each coordinate contains a point $\left\langle x_{1}, \ldots, x_{s}\right\rangle$ corresponding to a value of $k$ satisfying $0<k<m$, it also contains the point $\left\langle 1-x_{1}, \ldots\right.$, $\left.1-x_{s}\right\rangle$ obtained from the former by substituting $m-k$ for $k$. The point $\langle 0, \ldots, 0\rangle$ is in an exceptional situation, because the reduced lattice does not contain the point $\langle 1, \ldots, 1\rangle$, which would correspond to $k=m$. However, we can take care of it by replacing $f(0, \ldots, 0)$ by $2^{-1}\{f(0, \ldots, 0)+f(1, \ldots, 1)\}$. Thus we can replace the integrand by 


$$
\begin{array}{r}
F^{*}(\mathbf{x})=2^{1-s}\left\{f\left(x_{1}, \ldots, x_{s}\right)+f\left(1-x_{1}, x_{2}, \ldots, x_{s}\right)\right. \\
+f\left(x_{1}, 1-x_{2}, x_{3}, \ldots, x_{s}\right)+f\left(1-x_{1}, 1-x_{2}, x_{3}, \ldots, x_{s}\right) \\
\left.+\cdots+f\left(1-x_{1}, \ldots, 1-x_{s-1}, x_{s}\right)\right\}
\end{array}
$$

The effect of the simplification arising out of the use of $F^{*}$ instead of $F$ becomes even more important when, instead of a lattice generated by one lattice point $g$, we use a Cartesian product of lattices. It will be seen that such a procedure can be unexpectedly advantageous in some cases.

4. If we think of $Q^{s}$ as a Cartesian product $Q^{s^{\prime}} \times Q^{s^{\prime \prime}}$, we can represent $f$ as a function $f\left(\mathbf{x}^{\prime}, \mathbf{x}^{\prime \prime}\right)$ of an $s^{\prime}$-dimensional variable $\mathbf{x}^{\prime}$ and an $s^{\prime \prime}$-dimensional variable $\mathbf{x}^{\prime \prime}$. Having chosen good lattice points $\mathrm{g}^{\prime}$ modulo $m^{\prime}$ and $\mathrm{g}^{\prime \prime}$ modulo $m^{\prime \prime}$ in $s^{\prime}$ and $s^{\prime \prime}$ dimensions, respectively, we take

$$
\left(m^{\prime} m^{\prime \prime}\right)^{-1} \sum_{k^{\prime}=0}^{m^{\prime}-1} \sum_{k^{\prime \prime}=0}^{m^{\prime \prime}-1} f\left(m^{\prime-1} k^{\prime} \mathrm{g}^{\prime}, m^{\prime \prime-1} k^{\prime \prime} \mathrm{g}^{\prime \prime}\right)
$$

with all coordinates reduced modulo 1 , as an approximation of $\int_{Q^{s}} f(\mathbf{x}) d \mathbf{x}$. If $f$ is given by (3) and $c_{\mathrm{h}}$ satisfies (5), that is, if

$$
\left|c_{\mathrm{h}}\right| \leqslant K_{r} R\left(\mathrm{~h}^{\prime}\right)^{-(r+1)} R\left(\mathrm{~h}^{\prime \prime}\right)^{-(r+1)}
$$

where $h^{\prime}$ and $h^{\prime \prime}$ are the components of $h,(9)$ becomes

$$
\frac{1}{m^{\prime} m^{\prime \prime}} \sum_{\mathrm{h} \in \mathrm{Z}^{s}} c_{\mathrm{h}} \sum_{k^{\prime}=0}^{m^{\prime}-1} \sum_{k^{\prime \prime}=0}^{m^{\prime \prime}-1} \exp \left(2 \pi i\left(m^{\prime-1} k^{\prime} \mathrm{h}^{\prime} \cdot \mathrm{g}^{\prime}+m^{\prime \prime-1} k^{\prime \prime} \mathrm{h}^{\prime \prime} \cdot \mathrm{g}^{\prime \prime}\right)\right)
$$

and an upper bound of the absolute error of integration is obtained from this expression by subtracting the term in $c_{0}$ and substituting for $\left|c_{h}\right|$ its upper bound; the bound of the error is thus

$$
\begin{aligned}
K_{r} m^{\prime}-1 & \sum_{\mathbf{h} \in \mathbf{Z}^{s^{\prime}}} R\left(\mathbf{h}^{\prime}\right)^{-(r+1)} \sum_{k^{\prime}=0}^{m^{\prime}-1} \exp \left(2 \pi i m^{\prime-1} k^{\prime} \mathbf{h}^{\prime} \cdot \mathbf{g}^{\prime}\right) \\
& \times m^{\prime \prime-1} \sum_{\mathbf{h} \in \mathbf{Z}^{s^{\prime \prime}}} R\left(\mathbf{h}^{\prime \prime}\right)^{-(r+1)} \sum_{k^{\prime \prime}=0}^{m^{\prime \prime}-1} \exp \left(2 \pi i m^{\prime \prime-1} k^{\prime \prime} \mathbf{h}^{\prime \prime} \cdot \mathrm{g}^{\prime \prime}\right)-K_{r} \\
= & K_{r}\left\{\left(P^{(r+1)}\left(\mathrm{g}^{\prime}\right)+1\right)\left(P^{(r+1)}\left(\mathrm{g}^{\prime \prime}\right)+1\right)-1\right\} .
\end{aligned}
$$

This expression will be denoted by $K_{r} C_{2}^{(r+1)}\left(\mathrm{g}^{\prime}, \mathrm{g}^{\prime \prime}\right)$.

A similar argument applies to Cartesian products of more than two lattices. In particular, if we have three lattices generated by lattice points $\mathbf{g}^{\prime}, \mathbf{g}^{\prime \prime}$, and $\mathbf{g}^{\prime \prime \prime}$ in $s^{\prime}, s^{\prime \prime}$, and $s^{\prime \prime \prime}$ dimensions, respectively, $\left(s^{\prime}+s^{\prime \prime}+s^{\prime \prime \prime}=s\right)$, if the integrand satisfies the same conditions as before, and if we take as the approximate value of the integrand the average of $f$ over the Cartesian product of the lattices generated by $\mathbf{g}^{\prime}, \mathrm{g}^{\prime \prime}$, and $\mathrm{g}^{\prime \prime}$ and reduced modulo 1 in each coordinate, the error of integration is bounded in absolute value by

$$
K_{r} C_{3}^{(r+1)}\left(\mathrm{g}^{\prime}, \mathrm{g}^{\prime \prime}, \mathrm{g}^{\prime \prime \prime}\right)=K_{r}\left\{\left(P^{(r+1)}\left(\mathrm{g}^{\prime}\right)+1\right)\left(P^{(r+1)}\left(\mathrm{g}^{\prime \prime}\right)+1\right)\left(P^{(r+1)}\left(\mathrm{g}^{\prime \prime \prime}\right)+1\right)-1\right\}
$$

5. A special advantage of Cartesian products of good lattices arises when, in order to satisfy (ii) with $r=1$, we symmetrize the integrand as described in Section 3 . Each of the lattices in the product has the previously pointed out property of symmetry. 
As a result of it, we only have to replace the integrand by an average of $2^{s-2}$ values instead of $2^{s-1}$ if we had a product of two lattices, and of merely $2^{s-3}$ values if we had three lattices in the product. Of course, the value of $f$ at the origin has to be replaced by the obvious average of 4 or 8 values according to the number (two or three) of lattices in the Cartesian product.

This might still appear hardly useful since, by introducing a Cartesian product of two or three lattices, we lose a fair part of the general advantages of the method of good lattice points over iterated integration. However, this argument applies only to sufficiently large moduli, and "sufficiently large" in the context of large numbers of dimensions can be very large indeed. If the number of points over which we are willing to average the integrand is not "sufficiently large", a Cartesian product of two, or even three lattices may be more advantageous than a lattice generated by a single lattice point. Such a product is, of course, also a lattice; here and in what follows, by a lattice we mean a lattice of points reduced modulo 1 in each coordinate. We shall say that a lattice $L$ dominates a lattice $L^{\prime}$ in the same number of dimensions if the number of points of $L$ is smaller than that of $L^{\prime}$ and for $L$ the value of $P^{(2)}, C_{2}^{(2)}$, or $C_{3}^{(2)}$ according to the case is smaller than, or equal to, the relevant parameter of $L^{\prime}$.

6. Here are some systematically compiled examples of Cartesian products of lattices dominating good lattices generated by single lattice points. We compare lattices obtained in six to ten dimensions by A. I. Saltykov [5] with products of Fibonacci lattices in two dimensions and of lattices due to D. Maisonneuve [4] in three and four dimensions. In order to have a fair comparison, the values of $C_{2}^{(2)}$ and $C_{3}^{(2)}$ are rounded off to the number of decimals with which the corresponding $P^{(2)}$ was computed by D. Maisonneuve for Saltykov's lattices. We shall denote by $L\left(m ; g_{1}, \ldots, g_{s}\right)$ the lattice generated by $\mathrm{g}=\left\langle g_{1}, \ldots, g_{s}\right\rangle$ modulo $m$, and by $\times$ the Cartesian product of such lattices, squares being also understood as Cartesian products.

Six dimensions. $L(44 ; 1,14,20)^{2}$ (1936 points) with $C_{2}^{(2)}=1.9$ dominates

$$
L(2129 ; 1,41,1681,793,578,279) \quad \text { with } P^{(2)}=2.0 \text {. }
$$

Seven dimensions. $L(66 ; 1,10,24) \times L(266 ; 1,24,40,116)(17556$ points) with $C_{2}^{(2)}=1.05 \sim 1.1$ dominates

$$
L(18101 ; 1,17487,14976,44,9186,7308,1936) \quad \text { with } P^{(2)}=1.1 .
$$

Eight dimensions. $L(118 ; 1,18,40,52) \times L(168 ; 1,30,72,82)(19824$ points $)$ with $C_{2}^{(2)}=3.6$ dominates

$$
L(24041 ; 1,17441,21749,5411,12326,3144,21024,6252) \quad \text { with } P^{(2)}=3.9 \text {. }
$$

$L(168 ; 1,30,72,82)^{2}(28224$ points $)$ with $C_{2}^{(2)}=2.6$ and $L(180 ; 1,8,46,74)^{2}$ (32400 points) with $C_{2}^{(2)}=2.3$ dominate

$$
L(33139 ; 1,3520,29553,3239,1464,16735,19197,3019) \quad \text { with } P^{(2)}=2.7 \text {. }
$$

$L(266 ; 1,24,40,116)^{2}(70756$ points $)$ with $C_{2}^{(2)}=1.17 \sim 1.2$ dominates (weakly, though)

$$
L(71053 ; 1,60759,26413,24409,48215,51048,19876,29096) \quad \text { with } P^{(2)}=1.2 \text {. }
$$


Nine dimensions. $L(10 ; 1,6) \times L(26 ; 1,8,12) \times L(118 ; 1,18,40,52)(30680$ points) with $C_{3}^{(2)}=9.0$ dominates

$L(33139 ; 1,68,4624,16181,6721,26221,26661,23442,3384) \quad$ with $P^{(2)}=13.5$, and also,

$L(46213 ; 1,8871,40115,20065,30352,15654,42782,17966,33962)$

with $P^{(2)}=9.5$.

The latter is also dominated by $L(35 ; 1,11,16)^{3}$ (42875 points) with $C_{3}^{(2)}=8.4$.

$L(35 ; 1,11,16)^{2} \times L(44 ; 1,14,20)(53900$ points $)$ with $C_{3}^{(2)}=6.6$ dominates

$L(57091 ; 1,20176,12146,23124,2172,33475,5070,42339,36122)$

with $P^{(2)}=7.5$.

$L(35 ; 1,11,16) \times L(44 ; 1,14,20)^{2}(67760$ points $)$ with $C_{3}^{(2)}=5.1$ dominates $L(71053 ; 1,26454,13119,27174,17795,22805,43500,45665,49857)$

$L(44 ; 1,14,20)^{3}$ (85184 points) with $C_{3}^{(2)}=3.9$ dominates with $P^{(2)}=6.0$.

$L(100063 ; 1,70893,53211,12386,27873,56528,16417,17628,14997)$

with $P^{(2)}=4.1$.

Ten dimensions. Saltykov produced six good lattices; but one of them, of 145087 points with $P^{(2)}=15$, is dominated by his own lattice of 130703 points with $P^{(2)}=14$. Clearly, the former lattice can safely be ignored; its appearance in the table was obviously due to the fact that its author neither optimized nor computed $P^{(2)}(\mathrm{g})$ (see Section 2). Each of the remaining five lattices is dominated by at least two Cartesian products of three lattices. We show here only three such products, namely those which appear to be the most advantageous.

$L(26 ; 1,8,12)^{2} \times L(118 ; 1,18,40,52)\left(79768\right.$ points) with $C_{3}^{(2)}=14$ dominates Saltykov's lattices of $85633,103661,115069$, and 130703 points with $P^{(2)}=24$, 21,17 , and 14 , respectively.

$L(8 ; 1,5) \times L(118 ; 1,18,40,52)^{2}\left(111392\right.$ points) with $C_{3}^{(2)}=11$ dominates Saltykov's lattices of 115069,130703 , and 155093 points with $P^{(2)}=17,14$, and 12 , respectively. The last of these lattices is also dominated by

$$
L(10 ; 1,6) \times L(118 ; 1,18,40,52)^{2}(139240 \text { points }) \quad \text { with } C_{3}^{(2)}=9 \text {. }
$$

So far we did not take into account the advantages of Cartesian products of lattices which arise when the integrand has to be symmetrized. By way of example, it may be worth noting that if the integrand has to be symmetrized in at least three dimensions, the Cartesian product

$$
L(44 ; 1,14,20)^{2} \times L(266 ; 1,24,40,116)(514976 \text { points) }
$$

with $C_{3}^{(2)}=3.25679 \sim 3$ will require less computing work than Saltykov's lattice of 155093 points, while yielding an upper bound of the computing error which is about one-quarter of that which corresponds to the latter lattice.

Département de mathématiques

Université de Sherbrooke

Sherbrooke, Québec, Canada 
1. E. HLAWKA, “Zur angenäherten Berechnung mehrfacher Integrale," Monatsh. Math., v. 66, 1962, pp. 140-151. MR 26 \#888.

2. G. KEDEM \& S. K. ZAREMBA, "A table of good lattice points in three dimensions," Numer. Math., v. 23, 1974, pp. 175-180.

3. N. M. KOROBOV, Number-Theoretic Methods in Approximate Analysis, Fizmatgiz, Moscow, 1963. (Russian) MR 28 \#16.

4. DOMINIQUE MAISONNEUVE, "Recherche et utilisation des 'bons treillis'. Programmation et résultats numériques," Applications of Number Theory to Numerical Analysis, Academic Press, New York and London, 1972, pp. 121-201. MR 49 \#8270.

5. A. I. SALTYKOV, "Tables for evaluating multiple integrals by the method of optimal coefficients," Z. VyCisl. Mat. $i$ Mat. Fiz., v. 3, 1963, pp. 181-186=USSR Comput. Math. and Math. Phys., v. 3, 1963, pp. 235-242. MR 27 \#962.

6. S. K. ZAREMBA, "Good lattice points, discrepancy, and numerical integration," Ann. Mat. Pura Appl., v. 73 (4), 1966, pp. 293-317. MR 36 \#1107.

7. S. K. ZAREMBA, "La méthode des 'bons treillis' pour le calcul des intégrales multiples," Applications of Number Theory to Numerical Analysis, Academic Press, New York and London, 1972, pp. 39-119. MR 49 \#8271.

8. S. K. ZAREMBA, "Good lattice points modulo composite numbers," Monatsh. Math., v. 78, 1974, pp. 446-460.

9. S. K. ZAREMBA, "L'erreur dans le calcul des intégrales doubles par la méthode des bons treillis," Demonstratio Math., v. 8, 1975, pp. 347-364. 